\title{
AN ORDER THEORETIC CHARACTERIZATION OF NESTED SPACES
}

\author{
R. E. SMITHSON ${ }^{1}$
}

\begin{abstract}
A characterization of nested spaces in terms of their order theoretic properties is given. This characterization is analogous to the order theoretic characterization of trees given by $\mathbf{L}$. E. Ward, Jr.
\end{abstract}

Partial orders in topological spaces have been of great interest to topologists since the classic work of Nachbin [7]. Later L. E. Ward, Jr. [11] studied partially ordered topological spaces and in 1954 gave a characterization of trees in terms of an inherent partial order [12]. Recently Fugate, Gordh and Lum [1] and [2] gave an order theoretic characterization of arc smooth continua. Other similar results were obtained by Smithson [8], Smithson and Muenzenberger [4], and Ward [13].

Another area in which partial orders have played a key role is the study of dendritic spaces. See, in particular, the comprehensive work of Ward [14] and the recent results of Muenzenberger and Smithson [5] and [6]. In 1946, G. S. Young, Jr. [15] introduced the notion of a nested space. Nested spaces possess a natural order structure which has proven useful in obtaining fixed point theorems (see, for example, [9] and [10]). The algebraic properties of nested spaces were studied extensively by Muenzenberger and Smithson [3]. The purpose of the present paper is to give a characterization of nested spaces analogous to Ward's characterization of trees [12].

Suppose $(X, \leqslant)$ is a space with a partial order $\leqslant$. We set $L(x)=\{y: y<x\}$ and $M(x)=\{y: x \leqslant y\}$. A set $A \subset X$ is an antiset in case no two elements of $A$ are related. Further, the partial order is order dense in case $x<y$ implies that there is a $z \in X$ with $x<z<y$.

In this paper a continuum is a compact, connected $T_{2}$-space and an arc is a continuum which contains exactly two nonseparating points. A tree is a continuum in which any two distinct points can be separated by the omission of a third point. Then a space $X$ is nested in case it is arcwise connected, $T_{2}$ and the union of every nest of arcs is contained in an arc. If $X$ is a nested space and $a, b \in X$, then there is exactly one arc in $X$ with endpoints $a, b$. Using this fact we define a partial order $<$ on $X$ as follows. Let $e \in X$ be fixed. Then $x \leqslant y$ iff $x$ is an element of the arc with endpoints $e, y$. A number of properties of this partial order can be derived and

Received by the editors June 19, 1980 and, in revised form, October 30, 1980.

1980 Mathematics Subject Classification. Primary 54F05.

Key words and phrases. Partially ordered spaces, nested spaces.

${ }^{1}$ Presented to the American Mathematical Society January, 1980, in San Antonio, Texas, under the title Partial order structures in continua. 
some of these are given in the following theorem which is the order theoretic characterization of nested spaces mentioned above.

THeOREM. $A T_{2}$-space $X$ is a nested space if and only if $X$ admits a partial order $\leqslant$ which satisfies:

(1) There is a least element $e \in X$.

(2) $L(x)$ is compact and totally ordered for each $x \in X$.

(3) $\leqslant$ is order dense.

(4) If $A \subset X$ is an arc, then any antiset in $A$ contains at most two elements.

(5) Every maximal totally ordered subset of $M(a)$ is compact for all $a \in X$.

Proof. First suppose that $X$ is a nested space, and let $\leqslant$ be the order defined above. Then there is a least element $e$. Further, $L(x)$ is an arc (or $L(x)=\{e\}$ ) for each $x \in X$ and so (2) and (3) follow. Next, if $A \subset X$ is an arc, and if $x, y \in A$ are not related then $\inf \{x, y\}=x \wedge y=z$ exists and is in $A$. From this it follows that $A$ is the union of two totally ordered subarcs, one containing $x$ and the other $y$, and so (4) holds. Finally, (5) follows since the maximal totally ordered sets in $M(a)$ are arcs. Thus, a nested space admits a partial order satisfying the five conditions.

Now assume that $X$ admits a partial order $\leqslant$ which satisfies conditions (1)-(5). First, we shall show that if $A$ is a closed totally ordered set, then $M(x) \cap A$ is closed for all $x \in X$. We may assume that $M(x) \cap A \neq \varnothing$ and thus $M(x) \cap A$ is contained in a maximal totally ordered subset $A_{0}$ of $M(x)$ which is closed. But $M(x) \cap A=A \cap A_{0}$ and so $M(x) \cap A$ is closed. Next, let $a \in X$ and $x \in L(a)$. Then $M(x) \cap L(a)$ is closed. Thus the partial order restricted to the compact, $T_{2}$-space $L(a)$ satisfies: (i) $L(x), M(x)$ are closed in $L(a)$ for all $x \in L(a)$. (ii) $L(x) \cap L(y) \neq \varnothing$ and totally ordered for all $x, y \in L(a)$. (iii) It is order dense. (iv) $M(x)-x=L(a)-L(x)$ is open for all $x \in L(a)$. But these are exactly the conditions given by Ward [12] to characterize trees. Thus each $L(a)$ is an arc or the least element of the space, since each $L(a)$ is a totally ordered tree and it follows that $X$ is arcwise connected.

To show that $X$ is uniquely arcwise connected we shall use the following. If $A \subset X$ is an arc, then $M(x) \cap A$ is closed for each $x \in X$. To establish this, we first show that $A$ is the union of two closed totally ordered sets. If $A$ is totally ordered, then we are done. Thus, suppose there are noncomparable points $a_{1}$, $a_{2} \in A$. Let $A_{1}, A_{2}$ be maximal totally ordered subsets of $A$ which contain $a_{1}, a_{2}$, respectively. If $a_{3} \in A-\left(A_{1} \cup A_{2}\right)$, then there is a maximal totally ordered subset $A_{3}$ of $A$ with $a_{3} \in A_{3}$. Since $A_{i}$ is maximal, $L(x) \cap A \subset A_{i}$ whenever $x \in A_{i}$ for $i=1,2,3$. From this we deduce that the sets $A_{i}-\left(A_{j} \cup A_{k}\right), i, j, k$ distinct elements of $\{1,2,3\}$, are nonempty. But then if we pick $b_{1} \in A_{1}-\left(A_{2} \cup A_{3}\right)$, $b_{2} \in A_{2}-\left(A_{1} \cup A_{3}\right)$ and $b_{3} \in A_{3}-\left(A_{1} \cup A_{2}\right)$, we have an antiset with three elements, which is contrary to (4). Consequently, $A=A_{1} \cup A_{2}$. Then $(M(x) \cap A)^{*}$ $=\left(M(x) \cap A_{1}\right)^{*} \cup\left(M(x) \cap A_{2}\right)^{*}=\left(M(x) \cap A_{1}\right) \cup M(x) \cap A_{2}=M(x) \cap A$ and we are done.

Next if $A$ is any nonempty totally ordered set, then an application of (2) or (5) shows that the least upper bound and greatest lower bound of $A$ exist in $X$. 
Further, if $a, b$ are two noncomparable elements of $X$, then $L(a) \cap L(b)$ contains its least upper bound, which is the greatest lower bound of $a, b$. Therefore $a \wedge b=\operatorname{glb}\{a, b\}$ exists in $X$ for all $a, b \in X$.

Let $A \subset X$ be a nondegenerate arc and suppose $a, b$ are noncomparable elements of $A$. Then $a \wedge b \in A$. To see this we have from the above, $A=A_{1} \cup A_{2}$ where each $A_{i}$ is a closed totally ordered set. So each $A_{i}$ contains a largest element $a_{i}$. Then $A \subset L\left(a_{1}\right) \cup L\left(a_{2}\right)$ and suppose $a \in L\left(a_{1}\right)$ and $b \in L\left(a_{2}\right)$. Note that $a \wedge b=a_{1} \wedge a_{2}=c$. We may assume that $a_{1}, a_{2}$ are the endpoints of $A$, and since $L\left(a_{2}\right)$ is closed, there is a maximal half-open subarc of $A$ with endpoints $a_{1}, x_{0}$ which is contained in $L\left(a_{1}\right)-L\left(a_{2}\right)$. But then $x_{0} \in L\left(a_{1}\right) \cap L\left(a_{2}\right)$ and so $x_{0}<c$. Now all elements of the open arc from $a_{1}$ to $x_{0}$ in $A$ are in $M(c) \cap L\left(a_{1}\right)$ and if $x_{0}<c$ we obtain a contradiction to (5). Thus $x_{0}=c$ and so $c \in A$.

Now suppose that $X$ contains a circle $C$. If $C$ is totally ordered, it contains a largest element $x_{0}$ and then $C \subset L\left(x_{0}\right)$ which is a contradiction. Thus suppose $C$ contains two noncomparable elements $a, b$. Then $a, b$ are the endpoints of two arcs in $C$ and $a \wedge b$ must be in both of them, again a contradiction. Hence, we have shown that $X$ is uniquely arcwise connected.

Finally, we must show that the union of any nest of arcs is contained in an arc. For this note that any maximal totally ordered subset of a set $M(x)$ is an arc with one endpoint $x$. Further, if $A$ is an arc with endpoints $a, b$ with $a$ not related to $b$, then $A=A_{1} \cup A_{2}$ where $A_{1}=M(a \wedge b) \cap L(a), A_{2}=M(a \wedge b) \cap L(b)$ and $A_{1}, A_{2}$ are totally ordered subarcs. Thus let $\mathscr{Q}$ be a nest of arcs. If $\cup \mathscr{Q}$ is totally ordered we are done. Hence, suppose that $a_{1}, a_{2} \in \cup \mathbb{Q}$ and that $a_{1}, a_{2}$ are not related. Let $c=a_{1} \wedge a_{2}$. Let $a_{1}, a_{2} \in A_{0} \in \mathbb{Q}$, and $A \in \mathcal{Q}$. Since $A_{0} \subset A$ or $A \subset A_{0}$, it follows by the above that every element of $A$ is related to one of $a_{1}, a_{2}$. Thus define $A_{i}=\left\{x \in \cup \mathbb{Q}: x\right.$ is related to $\left.a_{i}\right\}$ for $i=1,2$. Then $\cup \mathbb{Q}=A_{1} \cup A_{2}$ and each of $A_{1}, A_{2}$ is totally ordered. Then $A_{i}$ is contained in an arc with one endpoint $c$ for each $i=1,2$, and the theorem is proved.

Now we present two examples of nonnested spaces which illustrate the significance of conditions (4) and (5).

EXAmple 1. Define the following sets in the plane: $X_{1}=\{(x, y): y=\sin \pi x$, $0<x \leqslant 1\} ; X_{2}=\{(x, y): x=0,-2 \leqslant y \leqslant 1\} ; X_{3}=\{(x, y): y=-2,0 \leqslant x \leqslant$ $1\} ; X_{4}=\{(x, y): x=1,-2 \leqslant y \leqslant 0\}$. Then $X=\cup_{i=1}^{4} X_{i}$ is a Warsaw circle. Let $e=(0,1)$ and $\leqslant$ the partial order previously defined with least element $e$. Then conditions (1)-(4) are all satisfied. However, $M(z)$ is totally ordered for all $z \in X$ but is closed only if $z=(0,1)$. Thus (5) fails. Note that the closure of each totally ordered set is totally ordered since $X$ is totally ordered. Further, if $A \subset X$ is an arc, then $A \cap M(x)$ is also an arc (or a point or empty) and so is closed. Thus (5) cannot be replaced by these weaker conditions.

Example 2. Let $X$ be the unit disk in the plane and let $e$ be the origin. Then $x \leqslant y$ in $X$ if and only if $x, y$ are in the same radial segment from $e$ and $x$ is between $e$ and $y$. Then conditions (1), (2), (3) and (5) are satisfied but $X$ contains arcs which are antisets. Hence, (4) fails.

These two examples show that (4) and (5) are independent and thus both are 
necessary in the theorem. Of course, there may be other statements which could replace either of them. An example of a nested space which is quite interesting is the following.

EXAmple 3. In the plane let $X_{n}=\{(1 / n, y): 0<y<1\}$ and let $X_{-n}=$ $\{(-1 / n, y):-1 \leqslant y \leqslant 0\}$ for $n \geqslant 2$. Let $X_{0}=\{(0, y):-1<y<1\}, X_{-1}=$ $\{(x,-1):-1 \leqslant x \leqslant 0\}$ and $X_{1}=\{(x, 1): 0 \leqslant x \leqslant 1\}$. Then $X=\cup_{n=-\infty}^{\infty} X_{n}$. It is easy to see that $X$ is nested. Moreover, there does not exist an $e$ so that the partial order with least element $e$ will have closed upper sets $M(x)$ for all $x \in X$. This is quite different from the situation for trees and arc smooth continua.

Fugate, Gordh and Lum [2] proved that a metric continuum $X$ is arc smooth if and only if $X$ admits a partial order which has a closed graph, which has a least element and for which the sets $L(x)$ are order arcs for each $x \in X$. From this and the above theorem we have:

COROLlary. A uniquely arcwise connected, arc smooth continuum is nested.

REMARK. The example above shows that the converse of this corollary does not hold.

Acknowledgement. The author wishes to thank Professor T. B. Muenzenberger, Kansas State University, for many useful discussions on this paper.

\section{REFERENCES}

1. J. B. Fugate, G. R. Gordh, Jr., and L. Lum, On arc smooth continua, Topology Proceedings 2 (1977).

2. _ Arc smooth continua, Trans. Amer. Math. Soc. (to appear).

3. T. B. Muenzenberger and R. E. Smithson, The structure of nested spaces, Trans. Amer. Math. Soc. 201 (1975), 57-87.

4. __ Mobs and semitrees, J. Aust. Math. Soc. 21 (1975), 56-63.

5. __ Semilattice structures on dendritic spaces, Topology Proceedings 2 (1977).

6. __ Fixed points for acyclic and dendritic spaces, Pacific J. Math. 72 (1977), 501-512.

7. L. Nachbin, Topology and order, Princeton Univ., Princeton, N. J., 1965.

8. R. E. Smithson, A note on acyclic continua, Colloq. Math. 19 (1968), 67-71.

9. , Fixed point theorems for certain classes of multifunctions, Proc. Amer. Math. Soc. 31 (1972), $595-600$.

10. A common fixed point theorem for nested spaces, Pacific J. Math. 82 (1979), 533-537.

11. L. E. Ward, Jr., Partially ordered topological spaces, Proc. Amer. Math. Soc. 5 (1954), 144-161.

12. ___ A note on dendrites and trees, Proc. Amer. Math. Soc. 5 (1954), 992-994.

13. __ Mobs, trees and fixed points, Proc. Amer. Math. Soc. 8 (1957), 798-804.

14. __ Recent developments in dendritic spaces and related topics, Studies in Topology (Proc. Conf. Univ. of North Carolina, Charlotte, N.C., March 14-16, 1974), Academic Press, New York, 1975, pp. 601-647.

15. G. S. Young, Jr., The introduction of local connectivity by change of topology, Amer. J. Math. 68 (1946), 479-494.

Department of Mathematics, University of Wyoming, Laramie, Wyoming 82071 